\title{
10 Jahre Technologietransfer- und Weiterbildungszentrum an der Technischen Fachhochschule Wildau e. V. (TWZ)
}

\author{
Heinz Kroske, Harald Zalejski
}

Am 12.09.1994 wurde das Technologietransfer- und Weiterbildungszentrum an der Technischen Fachhochschule Wildau in das Vereinsregister eingetragen. Das bedeutete den Abschluss einer langen, durch Prof. Dr.-Ing. Wilfried Arlt (Gründungsrektor und später Präsident der TFH Wildau) initiierten und organisierten Gründungsphase sowie den Beginn eines zielgerichteten Technologietransfers und einer auf die unterschiedlichen Formen der Weiterbildung orientierten Arbeit des TWZ e. V.

Seit Gründung der TFH Wildau wurde Technologietransfer als wichtige Aufgabe der Hochschule angesehen und viele Male wurde ein „Bus voll Professoren“ zusammen mit dem Rektor zu wichtigen Standorten von Industrie und Wirtschaft gefahren, um notwendige Kontakte für die in der Gründungsphase befindliche TFH Wildau zu knüpfen und Transferprojekte einzuwerben.

Bei dieser Einwerbung von Projekten und ihrer Realisierung wurde bald deutlich, dass es neben und an der Hochschule eine „Einrichtung“ geben müsste, die eigenständig agieren und Fördermöglichkeiten in Anspruch nehmen kann, die flexibler auf die Anforderungen der Wirtschaft reagieren und Hochschullehrern die Möglichkeit zum Aufbau von kleinen Instituten bieten kann.

Mögliche Formen dieser „Einrichtung“ wurden diskutiert und auch mit dem zuständigen Ministerium in Potsdam beraten. Als realisierbar stellte sich letztendlich die Gründung eines Vereins heraus.

In seiner Satzung wird der Zweck des Vereins formuliert. Er besteht in der

- Unterstützung des Technologietransfers zwischen Hochschule und Anwendern in der Praxis,

- Durchführung von Weiterbildungsveranstaltungen,

- Durchführung von Forschung und Entwicklung und

- Publizierung wissenschaftlicher Erkenntnisse.

Abgeleitet aus den Erfahrungen eines ersten Gründungsversuches im Frühjahr 1993 wurde als Prämisse für die Vereinsgründung auch eine möglichst überschaubare Mitgliederzahl gesetzt. So waren es lediglich sieben Mitglieder, die am 12. September 1994 das Technologie- und Weiterbildungszentrum an der TFH Wildau e. V. gründeten und ins Vereinsregister eintragen ließen: Professor Dr.-Ing. Wilfried Arlt, Professor Dr. Ernst Debusmann, Dr. Siegfried Görmer, Professor Dr.-Ing. habil. Bernd Hentschel, Dr. Bernd Reuter, Dr. Andreas Trupp und Dipl.-Ing. Harald Zalejski.

Erste Projekte ergaben sich aus der Kooperation mit Unternehmen der Region, wie z. B. NTBB Nachrichtentechnik GmbH Zeuthen, der Lutra Hafengesellschaft Königs
Wusterhausen, der SMB-Industrieholding GmbH Wildau, der Yacht- und Bootswerft Niederlehme, dem Stahlbau Niederlehme und der Schmidt/Lehmann GmbH Velten.

Bei den Kooperationsgesprächen zur Projekteinwerbung wurde bald deutlich, dass die wissenschaftliche Profilierung der beteiligten Hochschullehrer für die Kooperationspartner besonders wichtig ist. Engagierte Hochschullehrer erkannten den Vorteil der Gründung eines Instituts. Bereits im Studienführer 1993/94 werden als erste Institute genannt:

- Institut für Laser- und Plasmatechnik (Prof. Dr. Harald Gündel),

- Institut für Software und Organisation (Prof. Dr. Ernst Debusmann) und das

- Institut für Weiterbildung (Dipl.-Ing. Harald Zalejski).

Vom Institut für Weiterbildung wurden auch die ersten „Wildauer Workshops“ organisiert, so z. B. im September 1993 zum Thema „Qualitätsmanagement“ (Prof. Dr.-Ing. habil. Gerd Stegemann), im Oktober 1993 zum Thema „Altlastensanierung“ (Prof. Dr.-Ing. Udo Hellwig). Es folgten 1994 die Workshops „Produktionslogistik“, „Verkehrslogistik“, „Datenbanken“ und „Umweltmanagement”, um nur einige zu nennen.

Als erster Vereinsvorsitzender wurde Prof. Dr.-Ing. habil. Bernd Hentschel gewählt und als Vorstandsmitglieder Prof. Dr.-Ing. Wilfried Arlt und Dr. Bernd Reuter. Die für die Vereinsführung dringend notwendige Finanzarbeit wurde von Dr. Renate Wilde bewältigt.

Die Gründung des TWZ e. V. war und ist eng mit der TFH verbunden, ja sie ist ein Produkt der TFH, ein so genanntes An-Institut. Das kommt auch darin zum Ausdruck, dass zwischen TFH und TWZ e. V. ein Kooperationsvertrag erarbeitet und abgeschlossen wurde, in dem die Zusammenarbeit auf dem Gebiet von

- Forschung und Entwicklung,

- bei der Lehre und der Weiterbildung

- sowie in der internationalen Zusammenarbeit festgeschrieben ist.

Der Verein verfolgte von seiner Gründung an ausschließlich und unmittelbar gemeinnützige Zwecke. Die durch den Technologietransfer und die Weiterbildung erreichten Einnahmen und Ausgaben wurden in der Anfangszeit nach Projekten getrennt erfasst und zentral über das TWZ abgerechnet. Eine Zuordnung zu Instituten gab es noch nicht. Um die Tätigkeit der Projektleiter zu unterstützen und die Koordinierung im TWZ zu erleichtern, wurde es notwendig, Richtlinien zu erarbeiten. Sie sollten die Aufgabentei- 
lung zwischen Projektleiter und TWZ-Vorstand festlegen nach dem Motto „was darf der Projektleiter - was darf er nicht“. Daraus entstand dann 1995 die erste Fassung der Arbeitsgrundlagen und Richtlinien für die Projektbearbeitung im Technologietransfer- und Weiterbildungszentrum an der Technischen Fachhochschule Wildau e. V. In diesem Papier wurden die Aufgaben und Verantwortungsbereiche der Projektleiter definiert. In präzisierter Form gilt diese Richtlinie auch heute noch.

Zwischenzeitlich wurde ein immer stärkeres Interesse der Projektleiter deutlich, in der Öffentlichkeitsarbeit mit der Bezeichnung „Institutsleiter" zu operieren und damit ihre wissenschaftliche Profilierung deutlich darzustellen. Obwohl unsere Satzung weder in der alten, noch in der neueren Fassung Institute als Organisationsformen vorsah, wurde versucht - im Interesse einer aktiveren Transfertätigkeit - diesen Wünschen gerecht zu werden. Dabei musste berücksichtigt werden, dass ein im TWZ gebildetes Institut keine juristisch selbständige Institution darstellt, sondern lediglich eine zu Abrechnungszwecken intern gebildete Zusammenfassung von Unterkonten einzelner Projekte.

Diese Überlegungen fanden ihren Niederschlag in den Regelungen für den Geschäfts- und Schriftverkehr der Institute. Hier wurde unter anderem festgelegt, dass für den Schriftverkehr der Institute grundsätzlich Kopfbögen des TWZ e. V. verwendet werden und dass bei Angeboten bzw. Verträgen, d. h. allen Schriftstücken, die das TWZ Dritten gegenüber verpflichten, neben dem Institutsleiter der Vorstandsvorsitzende des TWZ e. V. zu unterschreiben hat.

Um die an der Errichtung eines Instituts Interessierten mit den daran geknüpften Voraussetzungen und den zu beachtenden Regelungen und Festlegungen vertraut zu machen, wurden die Erfahrungen aus der Arbeit mit Instituten in einer Vereinbarung zur Errichtung eines Instituts zusammengefasst. Diese Vereinbarungen stellen sozusagen die "Geburtsurkunde" für das Institut dar. Hier werden die zwischen dem TWZ-Vorstand und dem Institutsleiter getroffenen Festlegungen fixiert. Sie waren und sind auch heute noch die Grundlage für die Arbeit der Institute.

Seit Januar 1997 wird bei „Institutsgründungen“ nach diesen Regelungen verfahren. Die ersten auf dieser Basis errichteten Institute waren die Institute für

- Kreislaufwirtschaft (Prof. Dr. Gerhard Behrendt),

- Telekommunikation (Prof. Dr. Günter-Ulrich Tolkiehn),

- Kommunale Angelegenheiten (Prof. Dr. Götz Meder),

- Thermische Verfahrenstechnik (Prof. Dr. Josef Schmadl).

Heute werden TWZ-Projekte durch 20 Institute bearbeitet:

- China-Institut (Dr. Dirk Forschner) - seit 01. Januar 2005 gelöscht,

- Institut für Innovation und Energieverfahrenstechnik (Prof. Dr.-Ing. Udo Hellwig),

- Institut für Fernstudium (Prof. Dr. László Ungvári),

- Institut für Kreislaufwirtschaft (Prof. Dr. Gerhard Behrendt),

- Institut für Facility Management (Prof. Dr.-Ing. Dietrich Reiblich),

- Institut für Telekommunikation (Prof. Dr. Günter-Ulrich Tolkiehn),
- Institut für interkulturelle Kommunikation (Prof. Dr. Olga Rösch),

- Institut für Mittelstandsberatung (Prof. Dr. Peter Gadow),

- Institut für applied biology (Prof. Dr. Franz Wildenauer),

- Institut für Weiterbildung (Dr. Hartmut Stoltenberg),

- Institut für Mittelstand und Regionalentwicklung (Prof. Dr. Michael Büttner),

- Institut für Unternehmenslogistik (Prof. Dr.-Ing. habil. Bernd Hentschel),

- Institut für kommunale Angelegenheiten (Prof. Dr. Götz Meder),

- Institut für Wärmetechnik und thermische Stofftrennverfahren (Prof. Dr. Josef Schmadl),

- Institut für Plasma- und Lasertechnik (Prof. Dr. Sigurd Schrader),

- Institut für öffentliches Immobilienmanagement (Honorarprofessor Dipl.-Ing. Christian Killiches),

- Telematik-Institut für Software-Engineering (Prof. Dr. Ralf Vandenhouten),

- Institut für Regionalmanagement i. G. (Prof. Dr. Rainer Voß),

- Institut für Informatik in der Logistik i. G. (Prof. Dr. Stefan Bruntaler),

- Institut für Verkehrslogistik i. G. (Prof. Dr.-Ing. Herbert Sonntag).

Im Jahre 1997 gab es einen Wechsel in der Besetzung des Vorstandes. In der Mitgliederversammlung vom 26. Januar 1997 wurden in den Vorstand gewählt:

- Prof. Dr. Heinz Kroske (Vorsitzender),

- Dr. Bernd Reuter (1. Stellvertreter),

- Prof. Dr.-Ing. Wilfried Arlt (2. Stellvertreter),

- Dipl.-Ing. Harald Zalejski (Schatzmeister).

In den Folgejahren wurde versucht, eine personelle Verjüngung des Vereins und des Vorstandes vorzubereiten. Als 1. Stellvertreter wurde Prof. Dr. László Ungvári und als 2. Stellvertreter Prof. Dr. Siegfried Rolle in den Vorstand gewählt, nachdem die vorherigen Stellvertreter ausgeschieden waren.

Im Ergebnis dieser Bemühungen hat sich auch die Zahl der Vereinsmitglieder erhöht. Gegenwärtig besteht der Verein aus zwölf Mitgliedern. Prof. Dr.-Ing. Wilfried Arlt wurde vom Vorstand des TWZ anlässlich seiner Verabschiedung Ende des Jahres 1999 zum Ehrenmitglied ernannt.

\section{Ein kurzer Blick auf die Arbeitsergebnisse des Vereins}

Im Gründungsjahr 1994 wurden aus Workshops, Lehrgängen und Projekten Einnahmen von 122.300 DM erzielt. Im Jahre 1995 waren es bereits 245.700 DM. Maßgeblich an diesem Anstieg war das Projekt der Ausbildung von Meistern für Veranstaltungstechnik, das von 1995 bis 2000 vom Weiterbildungsinstitut betreut wurde.

Die im TZW e. V. insgesamt erzielten Erlöse aus Projekten erreichten im Jahre 1997 einen Spitzenwert von rund einer halben Million DM und haben sich in den letzten zwei Jahren auf etwa 200.000 Euro eingependelt. Dabei werden im Kalenderjahr etwa 25 bis 35 Projekte akquiriert, 
deren Leistungsumfang sich jeweils zwischen einigen hundert und mehreren zehntausend Euro bewegt. Aufgrund der Gemeinnützigkeit unseres Vereins muss dabei darauf geachtet werden, dass die Mehrheit der Projektinhalte dem Vereinszweck folgt, d. h. aus Weiterbildungsveranstaltungen oder ergebnisoffener Forschung stammt und damit den Anforderungen gemeinnütziger Tätigkeit entspricht.

Im Jahr 2003 erreichten die Umsätze der Institute nicht die Höhe, die eine Weiterexistenz des Vereins sichern konnten. Deshalb wurde durch die Mitglieder beschlossen, die Umlage von fünf auf acht Prozent zu erhöhen. In der darauf folgenden Diskussion wurde über die Möglichkeit beraten, alle Institute an der Finanzierung des Vereins zu beteiligen. Im Ergebnis der Diskussionen im Verein und mit den Institutsleitern wurde festgelegt, die Richtlinien zum Einrichten, Betreiben und zur Leitung eines Instituts neu zu fassen. Damit haben die Institute mit einer jährlichen Mindestzuführung zur Aufrechterhaltung der Vereinstätigkeit beizutragen.

Wenn man die bisherige Vereinsarbeit betrachtet, kann man Folgendes feststellen:

1. Das TWZ e. V. ist ein Produkt der Technischen Fachhochschule. Die enge Zusammenarbeit mit der Hochschule ist bis heute, jetzt unter dem Präsidenten Prof. Dr. László Ungvári, ein fester Grundsatz. Dazu gehört auch, dass die Präsidenten von Anfang an Vorstandsmitglieder waren und an der Gestaltung des Technologietransfers und der Vereinsarbeit aktiv mitwirkten.

2. Die interessierten Professoren und Angehörigen des Lehrkörpers gegebene Möglichkeit, für den Technologietransfer und die Weiterbildung im Rahmen des TWZ e. V. Institute zu gründen, wurde von Anbeginn genutzt und schlägt sich jetzt in der Anzahl von 19 Instituten nieder. Diese Entwicklung ist nur durch die Gründung des TWZ e. V. ermöglicht worden. Daher kann man die Institute auch - ganz profan ausgedrückt - als Kinder des TWZ e. V. betrachten.

3. Der Vorstand wirkt mit dem Ziel, die Institute, die auf Initiative von Angehörigen des Lehrkörpers gegründet worden sind, zu erhalten und zu fördern. Im Umkehrschluss heißt das auch: Um die Existenz der Institute zu sichern, muss der Verein bestehen bleiben. Er bietet Dienstleistungen für die Institute und benötigt finanzielle Mittel, um seine Tätigkeit durchführen zu können.

4. Um den Prozess der Gründung und die Erhaltung von Instituten einheitlich zu gestalten, hat der Vorstand in intensiver Arbeit Regularien für die Errichtung und das Betreiben eines Instituts festgelegt. Die jeweiligen Materialien wurden den Entwicklungsbedingungen des Vereins und des Technologietransfers angepasst und mehrfach überarbeitet. Diese Arbeit wurde im Wesentlichen von Dipl.-Ing. Harald Zalejski und Dr. Hartmut Stoltenberg geleistet.

Jetzt ist die „zweite Generation“ der Regularien gültig:

- die Vereinbarung zu Errichtung eines Instituts,

- die Richtlinien zur Errichtung und Betreiben eines Instituts und

- die Vereinbarung zur Leitung eines Instituts.

Der wesentliche Inhalt der Richtlinien besteht darin, dass über die Institute die Verbindungen zwischen der Hochschule und den Unternehmen, dem jeweiligen Forschungsgebiet entsprechend, kanalisiert werden. Ein Institut gewährt seinem jeweiligen Leiter die Anerkennung als qualifizierter Partner bei der Einwerbung von Projekten und demonstriert seine herausragende Stellung im Verhältnis zur Hochschule. Es erhöht damit das Prestige des Akquirierenden. Da das wiederum zur Beförderung des Technologietransfers beiträgt, kann diese Hauptzielsetzung des Vereins noch besser erfüllt werden.

5. Die Ausgaben des Vereins müssen niedrig gehalten werden. Sparsamkeit wird auch weiterhin einen wesentlichen Grundsatz der Vereinsarbeit bilden. Zur Aufrechterhaltung des TWZ e. V. ist es notwendig, dass mehr Institute Projekte einwerben und bearbeiten und damit die Existenz ihres Instituts und des Vereins sichern.

6. Mit dem Umfang der Projekte wächst auch die Möglichkeit, aus den Projektmitteln gegebenenfalls Mitarbeiter einzustellen.

Allen, die sich an dem Technologietransfer und an der Vereinsarbeit beteiligen, soll hier gedankt werden, und diejenigen, die Interesse an der Arbeit des TWZ e. V. haben, sind eingeladen sich einzubringen.

\section{Autoren}

Prof. Dr. Heinz Kroske

Havelländer Ring 63, 12629 Berlin

Tel. +49 3099902465

Dipl.-Ing. Harald Zalejski

Wildbahn 100 B, 15745 Wildau

Tel. +49 3375501536

zalejski@freenet.de 